\title{
Charm and charmonium-like spectroscopy in $B$ decays in Belle
}

\author{
T.A.-Kh. Aushev* \\ Swiss Federal Institute of Technology of Lausanne, Switzerland \\ Institute for Theoretical and Experimental Physics, Moscow, Russia
}

\begin{abstract}
We report measurements of the branching fractions for the decays $B \rightarrow D_{s 1}(2536)^{+} \bar{D}^{(*)}$, where $\bar{D}^{(*)}$ is $\bar{D}^{0}, D^{-}$or $D^{*-}$, and study of $B \rightarrow X(3872) K$ with $X(3872)$ decaying to $D^{* 0} \bar{D}^{0}$ using a sample of 657 million $B \bar{B}$ pairs recorded at the $\Upsilon(4 S)$ resonance with the Belle detector at the KEKB asymmetric-energy $e^{+} e^{-}$collider.
\end{abstract}

Key words: Charm, charmonium, exotic mesons, XYZ mesons

PACS: 14.40.Gx, 13.25.Hw, 12.39.Mk

\section{Introduction}

Recent discoveries of a number of new states, such as $D_{s 0}^{*}(2317), D_{s 1}(2460), X(3872)$, $Z$ (4430) and etc., show that our understanding of charm and charmonium spectroscopy might be incomplete. Quark Parton Model can not explain all of these states as $c \bar{s}$ or $c \bar{c}$ mesons. Some of them could be exotic hadrons including tetraquark mesons $(q \bar{q} q \bar{q})$ or molecule states. Study of the properties of these particles is important for the understanding of their structures.

The results presented in this article are based on a $605 \mathrm{fb}^{-1}$ data sample, corresponding to $657 \times 10^{6} B \bar{B}$ pairs, collected at the $\Upsilon(4 S)$ resonance with the Belle detector [1] at the KEKB asymmetric-energy $e^{+} e^{-}$collider [2].

\footnotetext{
* for the Belle Collaboration

Email address: aushev@itep.ru (T.A.-Kh. Aushev).
} 


\section{Study of the decays $B \rightarrow D_{s 1}(2536)^{+} \bar{D}^{(*)}$}

The $D_{s 1}(2536)^{+}$meson is reconstructed in its main decay modes: $D^{* 0}\left(D^{0} \pi^{0}\right) K^{+}$, $D^{* 0}\left(D^{0} \gamma\right) K^{+}$and $D^{*+} K_{S}$. The combinations of $D_{s 1}(2536)^{+}$and the second charm $D^{(*)}$ meson, which can be either $\bar{D}^{0}, D^{-}$or $D^{*-}$, with opposite flavor are considered as $B$ candidates. Inclusion of charge conjugate modes is implied throughout the paper.

The nine distributions corresponding to three $B$ decay modes times three $D_{s 1}(2536)^{+}$ decay modes are fitted simultaneously to obtain the branching fractions for each of the $B$ decay modes (Fig. 11). Statistically significant results are obtained for each of the decay modes.

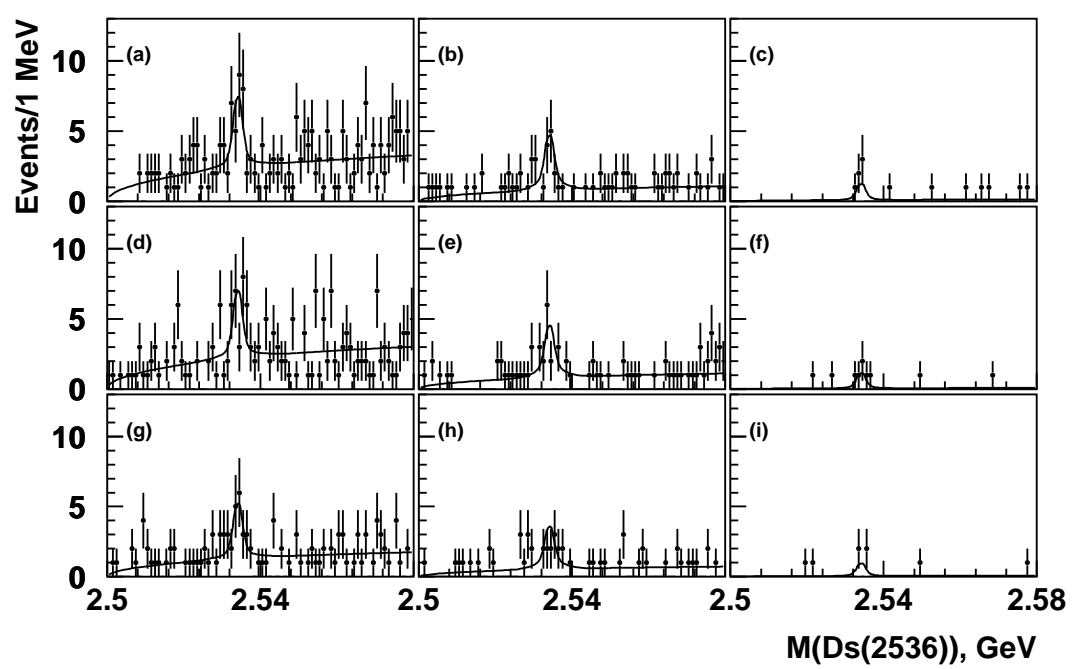

Fig. 1. $D_{s 1}^{+}(2536)$ mass distributions for the $B$ decays to a), b), c) $D_{s 1}^{+}(2536) \bar{D}^{0}$, d), e), f) $D_{s 1}^{+}(2536) D^{-}$ and g), h), i) $D_{s 1}^{+}(2536) D^{*-}$, followed by $D_{s 1}^{+}(2536)$ decays to a), d), g) $D_{s 1}^{+}(2536) \rightarrow D^{* 0}\left(D^{0} \gamma\right) K^{+}$, b), e), h) $D_{s 1}^{+}(2536) \rightarrow D^{* 0}\left(D^{0} \pi^{0}\right) K^{+}$and c), f), i) $D_{s 1}^{+}(2536) \rightarrow D^{*+}\left(D^{0} \pi^{+}\right) K_{S}$. Points with error bars show the data, lines represent the fit result.

Table 1

\begin{tabular}{lccc}
$B$ decay mode & $N$ & $\mathcal{B} \times 10^{4}$ & $\mathcal{S}$ \\
\hline$D_{s 1}(2536)\left(D^{*} K\right) \bar{D}^{0}$ & $42.7 \pm 8.6$ & $3.99 \pm 0.84 \pm 0.57$ & $7.0 \sigma$ \\
$D_{s 1}(2536)\left(D^{*} K\right) D^{-}$ & $40.4 \pm 8.7$ & $2.76 \pm 0.63 \pm 0.35$ & $6.9 \sigma$ \\
$D_{s 1}(2536)\left(D^{*} K\right) D^{*-}$ & $33.4 \pm 7.6$ & $5.03 \pm 1.21 \pm 0.68$ & $6.3 \sigma$
\end{tabular}

Summary of the results for $B \rightarrow D_{s 1}(2536)^{+} \bar{D}^{(*)}$ decay modes.

\section{Study of the decay $B \rightarrow X(3872)\left(D^{* 0} \bar{D}^{0}\right) K$}

The study described in this paper is a search for the $X(3872) \rightarrow D^{* 0} \bar{D}^{0}$ decay mode, followed either by $D^{* 0} \rightarrow D^{0} \gamma$ or $D^{* 0} \rightarrow D^{0} \pi^{0}$, in charged and neutral $B \rightarrow X(3872) K$ decays. We use the notation $D^{* 0} \bar{D}^{0}$ to indicate both $D^{* 0} \bar{D}^{0}$ and $\bar{D}^{* 0} D^{0}$. 
The $M\left(D^{*} D\right)$ mass distribution is described by a relativistic Breit-Wigner function convoluted with the mass-dependent Gaussian resolution for the signal and a square root function for the background. This fit gives $50.1_{-11.1}^{+14.8}$ events with a statistical significance of $8.8 \sigma$ (Fig. 2). The branching fraction, averaged over charged and neutral $B$ mesons, is

$$
\mathcal{B}(B \rightarrow X(3872) K) \times \mathcal{B}\left(X(3872) \rightarrow D^{* 0} \bar{D}^{0}\right)=(0.80 \pm 0.20 \pm 0.11) \times 10^{-4},
$$

where $\mathcal{B}\left(X(3872) \rightarrow D^{* 0} \bar{D}^{0}\right)$ stands for $\mathcal{B}\left(X(3872) \rightarrow D^{* 0} \bar{D}^{0}\right)+\mathcal{B}\left(X(3872) \rightarrow \bar{D}^{* 0} D^{0}\right)$.

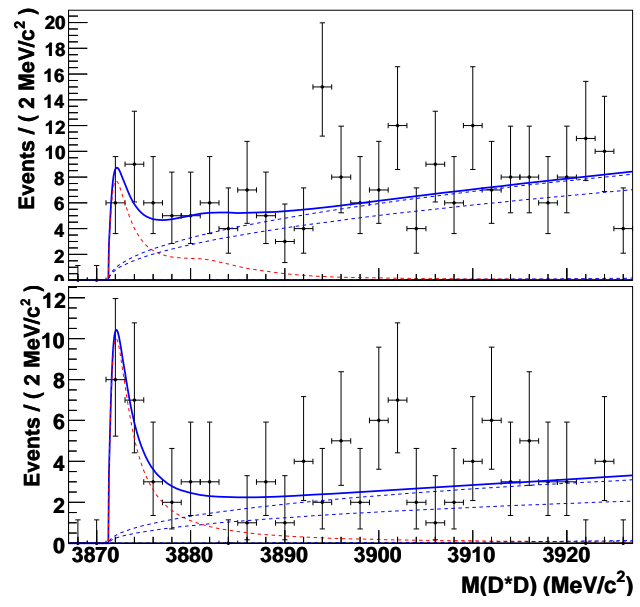

Fig. 2. Distribution of $M\left(D^{*} D\right)$ mass for $D^{* 0} \rightarrow D^{0} \gamma$ (top) and $D^{* 0} \rightarrow D^{0} \pi^{0}$ (bottom). The points with error bars are data, the dotted curve is the signal, the dashed curves represent the backgrounds.

\section{Conclusion}

In the summary we report the measurement of the branching fractions for the decays $B \rightarrow D_{s 1}(2536)^{+} \bar{D}^{(*)}$, where $\bar{D}^{(*)}$ is $\bar{D}^{0}, D^{-}$or $D^{*-}$. From the simultaneuos fit of all $D_{s 1}(2536)^{+}$channels we have measured $\mathcal{B}\left(B^{+} \rightarrow D_{s 1}(2536)^{+} \bar{D}^{0}\right)=(3.99 \pm 0.84 \pm 0.57) \times$ $10^{-4}, \mathcal{B}\left(B^{0} \rightarrow D_{s 1}(2536)^{+} D^{-}\right)=(2.76 \pm 0.63 \pm 0.35) \times 10^{-4}, \mathcal{B}\left(B^{0} \rightarrow D_{s 1}(2536)^{+} D^{*-}\right)=$ $(5.03 \pm 1.21 \pm 0.68) \times 10^{-4}$ with the statistical significances $7.0 \sigma, 6.9 \sigma$ and $6.3 \sigma$, respectivly.

We find a near-threshold enhancement in the $D^{* 0} \bar{D}^{0}$ invariant mass spectrum at $3872.9_{-0.4-0.4}^{+0.6+0.3} \mathrm{MeV} / c^{2}$ in $B \rightarrow D^{* 0} \bar{D}^{0} \mathrm{~K}$ decays. The significance of this enhancement is $8.8 \sigma$. The measured branching fraction of the decay is $\mathcal{B}(B \rightarrow X(3872) K) \times \mathcal{B}(X(3872) \rightarrow$ $\left.D^{* 0} \bar{D}^{0}\right)=(0.80 \pm 0.20 \pm 0.11) \times 10^{-4}$.

\section{References}

[1] Belle Collaboration, A. Abashian et al., Nucl. Instr. and Meth. A479, 117-232 (2002).

[2] KEKB B Factory Design Report, KEK Report 95-1, 1995, unpublished. 